PROCEEDINGS OF THE

AMERICAN MATHEMATICAL SOCIETY

Volume 132, Number 9, Pages 2793-2797

S 0002-9939(04)07396-4

Article electronically published on March 2, 2004

\title{
INDECOMPOSABLES OF MULTIPLICATIVE FIBRATIONS
}

\author{
JAMES P. LIN
}

(Communicated by Paul Goerss)

\begin{abstract}
Given a multiplicative fibration $F \stackrel{j}{\longrightarrow} E \stackrel{\pi}{\longrightarrow} B$ we study the module of indecomposables $Q H^{*}\left(E ; \mathbb{Z}_{p}\right)$ for $p$ a prime.
\end{abstract}

A fibration $F \stackrel{j}{\longrightarrow} E \stackrel{\pi}{\longrightarrow} B$ is a multiplicative fibration if $E$ and $B$ are connected $H$-spaces and $\pi: E \longrightarrow B$ is an $H$-map. In this note we study the module of indecomposables $Q H^{*}\left(E ; \mathbb{Z}_{p}\right)$ for $p$ a prime.

Theorem 0.1 (Main Theorem). Let $F \stackrel{j}{\longrightarrow} E \stackrel{\pi}{\longrightarrow} B$ be a multiplicative fibration.

(a) Let $p$ be an odd prime. If $n \not \equiv 0 \bmod 2 p$ and $n \not \equiv \pm 1 \bmod 2 p$, then there are exact sequences

$$
\begin{aligned}
& P H_{n}\left(F ; \mathbb{Z}_{p}\right) \stackrel{P j_{*}}{\longrightarrow} P H_{n}\left(E ; \mathbb{Z}_{p}\right) \stackrel{P \pi_{*}}{\longrightarrow} P H_{n}\left(B ; \mathbb{Z}_{p}\right), \\
& Q H^{n}\left(F ; \mathbb{Z}_{p}\right) \stackrel{Q j^{*}}{\longleftarrow} Q H^{n}\left(E ; \mathbb{Z}_{p}\right) \stackrel{Q \pi^{*}}{\longleftarrow} Q H^{n}\left(B ; \mathbb{Z}_{p}\right) .
\end{aligned}
$$

(b) Let $n \equiv 0 \bmod 2 p$ for $p$ odd or $n$ even for $p=2$. Suppose

$$
Q H^{n}\left(F ; \mathbb{Z}_{p}\right) \oplus \sum_{k=1}^{\infty} Q^{n}\left(\xi^{k} H^{*}\left(F ; \mathbb{Z}_{p}\right)\right)=0
$$

where $\xi: H^{*}\left(F ; \mathbb{Z}_{p}\right) \longrightarrow H^{*}\left(F ; \mathbb{Z}_{p}\right)$ is the pth power map. Then $Q H^{n}\left(E ; \mathbb{Z}_{p}\right)=$ $Q \pi^{*} Q H^{n}\left(B ; \mathbb{Z}_{p}\right)$.

Theorems similar to the Main Theorem have been proved under more specialized assumptions. For example, if $H^{*}\left(E ; \mathbb{Z}_{p}\right)$ is a $U(M)$ module, such multiplicative fibrations were studied by Massey and Peterson [6, 7. In the case of loop fibrations, these sequences have been considered by Goerss, Lannes and Morel 2]. Moore and Smith also study multiplicative fibrations using the Eilenberg-Moore spectral sequence [10. We will use this theorem in a future paper to investigate the cohomology of finite $H$-spaces with nontrivial Steenrod action on the even degree indecomposables. As a corollary of the Main Theorem, we make some observations about the ring structure of $H_{*}\left(E ; \mathbb{Z}_{p}\right)$.

We assume all spaces have the homotopy type of path connected $\mathrm{CW}$ complexes with basepoint and all homology and cohomology modules are finitely generated.

Received by the editors May 1, 2003 and, in revised form, May 28, 2003.

2000 Mathematics Subject Classification. Primary 55R20, 55R35, 55R05.

Key words and phrases. $H$-space, multiplicative fibration, Serre spectral sequence.

(C)2004 American Mathematical Society 
The ground field will be the field $\mathbb{Z}_{p}$ of $p$ elements where $p$ is a prime. Given a connected Hopf algebra $A, I(A)$ will denote the elements of $A$ of positive degree. $P(A)$ and $Q(A)$ will denote the primitives and indecomposables respectively. $\alpha_{A}$ : $I(A) \longrightarrow Q(A)=I(A) / I(A)^{2}$ will denote the projection map.

The author would like to thank John McCleary for several useful comments. Some of the work for this paper was done while visiting Yutaka and Keiko Hemmi at Kochi University in Kochi, Japan. The author wishes to thank them for their wonderful hospitality during his stay. The author also thanks the referee for several useful comments.

Proposition 0.2. Let $A$ and $B$ be connected commutative Hopf algebras over $\mathbb{Z}_{p}$ with $B$ a sub-Hopf algebra of $A$.

(a) Let $n \not \equiv 0 \bmod 2 p$, for $p$ odd or $n$ odd for $p=2$. Then the inclusion map $i: B \longrightarrow A$ induces a monomorphism $Q i: Q^{n} B \longrightarrow Q^{n} A$.

(b) Let $n \equiv 0 \bmod 2 p$ for $p$ odd or $n$ even for $p=2$. Suppose $0 \neq \alpha_{B}(b) \in$ $Q^{n} B \cap \operatorname{ker} Q i$. Then $\alpha_{B}(b)$ can be represented by a $p^{k}$ th power of an algebra generator $a \in A$ for some $k \geq 1$.

Proof. Suppose $0 \neq \alpha_{B}(b) \in Q^{n}(B) \cap \operatorname{ker} Q i$. Let $B(n-1)$ be the sub-Hopf algebra of $B$ generated by elements of $B$ of degree less than $n$. We have a commutative diagram

$$
\begin{array}{lll}
B & \stackrel{\pi_{B}}{\longrightarrow} & B / / B(n-1) \\
i \downarrow & & \downarrow \theta \\
A & \stackrel{\pi_{A}}{\longrightarrow} & A / / B(n-1)
\end{array}
$$

where $\pi_{B}, \pi_{A}$ are Hopf algebra epimorphisms and $0 \neq \pi_{B}(b) \in P^{n}(B / / B(n-1)) \cong$ $Q^{n} B$. By [3, p. 8] there exist isomorphisms $B \cong B(n-1) \otimes B / / B(n-1)$ and $A \cong B(n-1) \otimes A / / B(n-1)$ such that the following diagram commutes:

$$
\begin{gathered}
B \cong B(n-1) \otimes B / / B(n-1) \\
\downarrow 1 \otimes \theta \\
i \downarrow \\
A \cong B(n-1) \otimes A / / B(n-1)
\end{gathered}
$$

Therefore, $\theta$ is a monomorphism.

We have a commutative diagram:

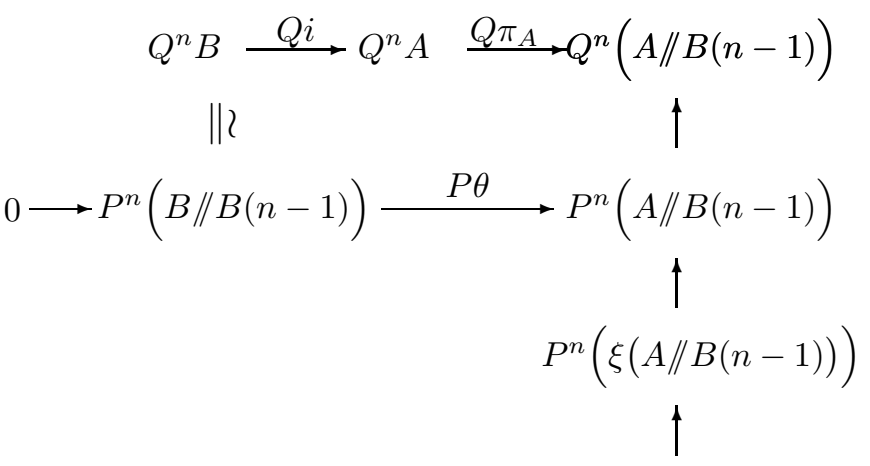

0

The bottom row is exact since $\theta$ is monic. The right column is exact by 9 Proposition 4.2.21]. Now given $\operatorname{Qi\alpha }_{B}(b)=0$, by diagram (0.1), it follows that 
$\pi_{A}(b) \in P^{n}(A / / B(n-1))$ is decomposable. By exactness of the right column of (0.1), $\pi_{A}(b)$ is a $p$ th power. In case (a) $n \not \equiv 0 \bmod 2 p$; this is impossible and $Q i: Q^{n} B \rightarrow Q^{n} A$ is a monomorphism. In case (b) $n \equiv 0 \bmod 2 p$ for $p$ odd or $n$ is even for $p=2$. For each $k \geq 1$ there are exact sequences [9. Proposition 4.2.21]

$$
0 \longrightarrow P\left(\xi^{k+1}(A / / B(n-1))\right) \longrightarrow P\left(\xi^{k}(A / / B(n-1))\right) \longrightarrow Q\left(\xi^{k}(A / / B(n-1))\right) \text {. }
$$

By using these sequences we can show that $\alpha_{B}(b)$ can be represented by a $p^{k}$ th power of a generator of $A$ for some $k \geq 1$. This proves (b).

Let $F \stackrel{j}{\longrightarrow} E \stackrel{\pi}{\longrightarrow} B$ be a multiplicative fibration. By [1, Lemma 5.1], $\pi_{1}(B)$ acts trivially on $H_{*}\left(F ; \mathbb{Z}_{p}\right)$. Hence the Serre spectral sequence for the fibration satisfies

$$
E_{2}^{r, s}=H^{r}\left(B ; \mathbb{Z}_{p}\right) \otimes H^{s}\left(F ; \mathbb{Z}_{p}\right)
$$

and

$$
E_{\infty} \cong \mathcal{G}_{r} H^{*}\left(E ; \mathbb{Z}_{p}\right)
$$

This is a first quadrant spectral sequence of Hopf algebras [1 p. 166]. Furthermore, by [1, Theorem 5.8],

$$
E_{k} \cong B_{k} \otimes C_{k} \otimes M_{k} \otimes N_{k} \quad \text { for } \quad k \geq 2 \quad \text { as algebras }
$$

where

$$
B_{k}=E_{k}^{0, *} \text { and } C_{k}=E_{k}^{*, 0} \text { are sub-Hopf algebras of } E_{k} .
$$

$M_{k}=\Lambda\left(x_{1}, \ldots, x_{\ell}\right)$ and $N_{k}=\Lambda\left(w_{1}, \ldots, w_{n}\right)$ with $\operatorname{dim} x_{i} \equiv 1 \bmod 2 p$ and $\operatorname{dim} w_{i} \equiv$ $-1 \bmod 2 p$. Equation (0.3) implies

$$
Q E_{\infty}^{r, s}=0 \text { for } r>0 \text { and } s>0 \text { and } r+s \not \equiv \pm 1 \bmod 2 p .
$$

Hence, if $n \not \equiv \pm 1 \bmod 2 p$,

$$
Q^{n} E_{\infty}=Q E_{\infty}^{0, n} \oplus Q E_{\infty}^{n, 0} .
$$

By [8, Theorem 5.8], $j^{*}: H^{n}\left(E ; \mathbb{Z}_{p}\right) \longrightarrow H^{n}\left(F ; \mathbb{Z}_{p}\right)$ is the composition

$$
H^{n}\left(E ; \mathbb{Z}_{p}\right) \longrightarrow E_{\infty}^{0, n}=E_{n+1}^{0, n} \subset \cdots \subset E_{2}^{0, n}=H^{n}\left(F ; \mathbb{Z}_{p}\right),
$$

and $\pi^{*}: H^{n}\left(B ; \mathbb{Z}_{p}\right) \longrightarrow H^{n}\left(E ; \mathbb{Z}_{p}\right)$ is the composition

$$
H^{n}\left(B ; \mathbb{Z}_{p}\right)=E_{2}^{n, 0} \longrightarrow E_{n+1}^{n, 0}=E_{\infty}^{n, 0} \subseteq H^{n}\left(E ; \mathbb{Z}_{p}\right) .
$$

Proof of the Main Theorem 0.1 . The sequences of (a) are dual. So it suffices to prove

$$
Q H^{n}\left(B ; \mathbb{Z}_{p}\right) \stackrel{Q \pi^{*}}{\longrightarrow} Q H^{n}\left(E ; \mathbb{Z}_{p}\right) \stackrel{Q j *}{\longrightarrow} Q H^{n}\left(F ; \mathbb{Z}_{p}\right)
$$

is exact if $p$ is an odd prime, $n \not \equiv 0 \bmod 2 p$ and $n \not \equiv \pm 1 \bmod 2 p$. Note that $\pi j$ is null homotopic. Therefore, $Q j^{*} Q \pi^{*}=0$. So it suffices to prove $\operatorname{ker} Q j^{*} \subseteq \operatorname{im} Q \pi^{*}$.

Let $\alpha: I H^{*}\left(E ; \mathbb{Z}_{p}\right) \longrightarrow Q H^{*}\left(E ; \mathbb{Z}_{p}\right)$ be the projection. Let $\alpha(w) \in \operatorname{ker} Q j^{*} \cap$ $Q H^{n}\left(E ; \mathbb{Z}_{p}\right)$. The Serre spectral sequence is a spectral sequence of algebras. Hence, $w$ must produce a nonzero element of $Q^{n} E_{\infty}$. By (0.5),

$$
Q^{n} E_{\infty}=Q E_{\infty}^{0, n} \oplus Q E_{\infty}^{n, 0} .
$$

Suppose $\alpha(w)$ has a nonzero component in $Q E_{\infty}^{0, n}$. By $(0.3)$ and $\left([0.6), E_{\infty}^{0, *}\right.$ is a sub-Hopf algebra of $H^{*}\left(F ; \mathbb{Z}_{p}\right)$. By Proposition 0.2 (a), since $n \neq \equiv \bmod 2 p$, and $p$ is odd, a nonzero element of $Q E_{\infty}^{0, n}$ produces a nonzero element of $Q H^{n}\left(F ; \mathbb{Z}_{p}\right)$. 
By ([0.6), this would imply that $Q j^{*}(\alpha(w)) \neq 0$. But $\alpha(w) \in \operatorname{ker} Q j^{*}$. Therefore, $\alpha(w)$ has zero component in $Q E_{\infty}^{0, n}$ and $\alpha(w)$ lies in $Q E_{\infty}^{n, 0}$. By (0.7),

$$
H^{n}\left(B ; \mathbb{Z}_{p}\right) \longrightarrow E_{\infty}^{n, 0} \quad \text { is onto. }
$$

Hence $\alpha(w)$ lies in the image of $Q \pi^{*}$. This proves (a). To prove (b) let $\alpha(w) \in$ $Q H^{n}\left(E ; \mathbb{Z}_{p}\right)$ with $n \equiv 0 \bmod 2 p$ for $p$ odd or $n$ even for $p=2$. Suppose $\alpha(w)$ has nonzero component $x$ in $Q E_{\infty}^{0, n}$. Since $Q H^{n}\left(F ; \mathbb{Z}_{p}\right)=0$, the inclusion map induces the trivial map

$$
Q i: Q E_{\infty}^{0, n} \longrightarrow Q H^{n}\left(F ; \mathbb{Z}_{p}\right) \text {. }
$$

By Proposition 0.2 (b), $x$ can be represented by a $p^{k}$ th power of an algebra generator of $H^{*}\left(F ; \mathbb{Z}_{p}\right)$. But $Q^{n}\left(\xi^{k} H^{*}\left(F ; \mathbb{Z}_{p}\right)\right)=0$. Hence $\alpha(w)$ has no component in $Q E_{\infty}^{0, n}$ and $\alpha(w)$ lies in $Q E_{\infty}^{n, 0}$. By (0.8), $\alpha(w)$ lies in the image of $Q \pi^{*}$.

Let $f: B \rightarrow K\left(\mathbb{Z}_{p}, \ell+1\right)$ be an $H$-map, and let $E$ be the fibre of $f$. We have the following multiplicative fibration:

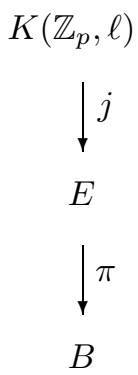

In general, the algebra structure of $H_{*}\left(E ; \mathbb{Z}_{p}\right)$ is difficult to compute. We can, however, make the following observations.

Corollary 0.3. Let $p$ be an odd prime. Let $s, t \in P H_{*}\left(E ; \mathbb{Z}_{p}\right)$ with $\pi_{*}(s) \neq$ $0, \pi_{*}(t) \neq 0$, and $\left[\pi_{*}(s), \pi_{*}(t)\right]=0$. Suppose $\operatorname{deg}[s, t]=n$ and $n \not \equiv \pm 1 \bmod 2 p$ and $n \not \equiv 0 \bmod 2 p$. If $Q H^{n}\left(K\left(\mathbb{Z}_{p}, \ell\right) ; \mathbb{Z}_{p}\right)=0$, then $[s, t]=0$.

Proof. By the Main Theorem 0.1 (a), since $P H_{n}\left(K\left(\mathbb{Z}_{p}, \ell\right) ; \mathbb{Z}_{p}\right)=0$, if $[s, t] \neq 0$, then $\pi_{*}[s, t]=\left[\pi_{*}(s), \pi_{*}(t)\right] \neq 0$. We conclude $[s, t]=0$.

Corollary 0.4. Let $t \in P H_{2 m}\left(E ; \mathbb{Z}_{p}\right)$ with $\pi_{*}(t) \neq 0$, and $\left(\pi_{*}(t)\right)^{p}=0$. If $Q H^{2 m p}\left(K\left(\mathbb{Z}_{p}, \ell\right) ; \mathbb{Z}_{p}\right) \oplus \sum_{k=1}^{\infty} Q^{2 m p}\left(\xi^{k} H^{*}\left(K\left(\mathbb{Z}_{p}, \ell\right) ; \mathbb{Z}_{p}\right)\right)=0$, then $t^{p}=0$.

Proof. If $t^{p} \neq 0$, there exists an indecomposable $\gamma \in Q H^{2 m p}\left(E ; \mathbb{Z}_{p}\right)$ with $\left\langle t^{p}, \gamma\right\rangle=1$. By the Main Theorem 0.1 (b), $\gamma=\pi^{*}\left(\gamma_{1}\right)+d$ where $\gamma_{1}$ is indecomposable and $d$ is decomposable. Then $1=\left\langle t^{p}, \gamma\right\rangle=\left\langle t^{p}, \pi^{*}\left(\gamma_{1}\right)+d\right\rangle=\left\langle t^{p}, \pi^{*}\left(\gamma_{1}\right)\right\rangle=\left\langle\left(\pi_{*}(t)\right)^{p}, \gamma_{1}\right\rangle=$ 0 . This is a contradiction. Therefore, $t^{p}=0$.

\section{REFERENCES}

[1] W. Browder, On Differential Hopf Algebras, Trans. Amer. Math. Soc. 107 (1963), 153-176. MR 26:3061

[2] P. Goerss, J. Lannes and F. Morel, Vecteurs de Witt non commutatifs et représentabilité de l'homologie modulo p, Inventiones Mathematicae 108 (1992), 163-227. MR 93e:55014]

[3] R. Kane, The Homology of Hopf Spaces, North-Holland, Amsterdam, 1988. MR 90f:55018 
[4] J. P. Lin, $H$-spaces analogous to $E_{8} \bmod 3$, Math. Zeit., to appear.

[5] J. P. Lin, Torsion in H-spaces I, Ann. Math. 103 (1976), 457-487. MR 54:13911

[6] W. S. Massey and F. P. Peterson, The cohomology structure of certain fibre spaces I, Topology 4 (1965), 47-65. MR 32:6459

[7] W. S. Massey and F. P. Peterson, The mod 2 cohomology structure of certain fibre spaces, Amer. Math. Soc. Memoirs 74 (1967). MR 37:2226

[8] J. McCleary, User's Guide to Spectral Sequences, Mathematical Lecture Series 12, Publish or Perish, Inc., 1985. MR 87f:55014

[9] J. Milnor and J. C. Moore, On the Structure of Hopf algebras, Ann. Math. 81 (1965), 211-264. MR 30:4259

[10] J. C. Moore and L. Smith, Hopf algebras and multiplicative fibrations I, II, Amer. J. Math. 90 (1968), 752-780, 1113-1150. MR 38:2772] MR 38:6599

Department of Mathematics, University of California, San Diego, La Jolla, CaliFORNIA 92093-0112

E-mail address: jimlin@euclid.ucsd.edu 